\title{
Avaliação do desgaste do polietileno de uma prótese de joelho nacional ultracongruente de base rotatória*
}

\section{Evaluation of Polyethylene Wear in a Brazilian Ultracongruent Knee Prosthesis with a Rotating Platform}

\author{
José Ricardo Pécora ${ }^{1}$ Valéria Romero 2 (1) \\ ${ }^{1}$ Grupo do Joelho, Departamento de Ortopedia e Traumatologia, \\ Instituto de Ortopedia e Traumatologia, Hospital das Clínicas, \\ Faculdade de Medicina, Universidade de São Paulo (HCFMUSP), \\ São Paulo, SP, Brasil \\ ${ }^{2}$ Faculdade de Medicina, Universidade de Campinas, Campinas, SP, Brasil \\ Rev Bras Ortop 2021;56(1):42-46
}

\begin{abstract}
Endereço para correspondência Valéria Romero, PhD, Faculdade de Medicina, Universidade de Campinas, Rua Sérgio Buarque de Holanda, Cidade Universitária, Campinas, SP, 13083859, Brasil (e-mail: emaildavaleria@gmail.com).
\end{abstract}

\section{Resumo \\ Palavras-chave \\ - artroplastia do joelho \\ - prótese do joelho \\ - desenho de prótese \\ - falha de prótese}

\section{Abstract}

Objetivo Avaliar o desgaste do polietileno de uma prótese de joelho brasileira ultracongruente de base rotatória (Rotaflex, Víncula, Rio Claro, SP, Brasil).

Métodos Utilizou-se o método de ensaio com os parâmetros de carregamento e preparação citados na norma ISO 14243-1:2009, e os métodos de medição citados na norma ISO 14243-2:2009, para a avaliação do comportamento de desgaste de uma prótese nacional com base rotatória. O equipamento utilizado para o teste de desgaste foi o simulador de marcha ISO 14243-1 (EndoLab, Riedering, Alemanha).

Resultados Após 10 milhões de ciclos, a avaliação do desgaste do polietileno mostrou uma aparência regular do desgaste da superfície com taxa média de $2,56 \mathrm{mg}$ por milhão de ciclos.

Conclusão O desgaste do polietileno da prótese avaliada foi mínimo após os ensaios realizados e com os limites de segurança superiores aos preconizados pela engenharia biomecânica.

Objective To evaluate the wear of polyethylene in a Brazilian ultracongruent knee prosthesis with a rotating platform (Rotaflex, Víncula, Rio Claro, SP, Brazil).

Methods We used the test method with the loading and preparation parameters mentioned in the standards regulation ISO 14243-1:2009, and the measurement methods

\footnotetext{
Trabalho desenvolvido no Grupo do Joelho, Departamento de Ortopedia e Traumatologia, Hospital das Clínicas, Faculdade de Medicina, Universidade de São Paulo (HCFMUSP), São Paulo, SP, Brasil, e na Faculdade de Medicina, Universidade de Campinas, Campinas, SP, Brasil.
}

recebido

04 de Fevereiro de 2020

aceito

02 de Março de 2020

Publicado online

Junho 10, 2020
DOI https://doi.org/ $10.1055 / \mathrm{s}-0040-1712491$. ISSN $0102-3616$

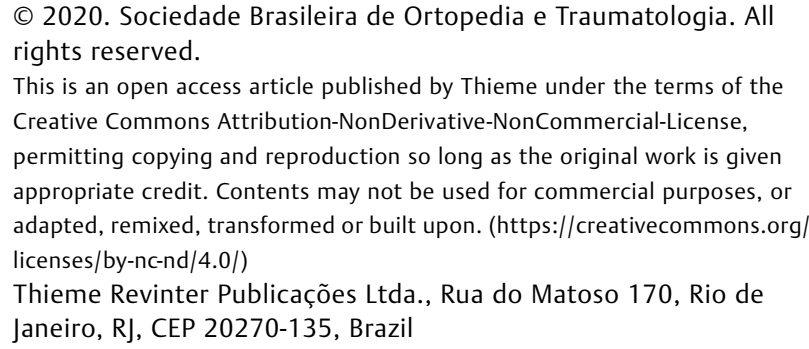

(c) 2020. Sociedade Brasileira de Ortopedia e Traumatologia. All rights reserved.

This is an open access article published by Thieme under the terms of the Creative Commons Attribution-NonDerivative-NonCommercial-License, permitting copying and reproduction so long as the original work is given appropriate credit. Contents may not be used for commercial purposes, or adapted, remixed, transformed or built upon. (https://creativecommons.org/ licenses/by-nc-nd/4.0/) Thieme Revinter Publicações Ltda., Rua do Matoso 170, Rio de Janeiro, RJ, CEP 20270-135, Brazil 


\section{Keywords}

- arthroplasty, replacement, knee

- knee prosthesis

- prosthesis design

- prosthesis failure mentioned in the standards regulation ISO 14243-2:2009, for the evaluation of the wear behavior of a Brazilian prosthesis with a rotating platform. The equipment used for the wear test was the ISO 14243-1 gait simulator (EndoLab, Riedering, Germany).

Results After 10 million cycles, the evaluation of the polyethylene wear showed a regular appearance of surface wear at a mean rate of $2.56 \mathrm{mg}$ per million cycles.

Conclusion The wear of the polyethylene of the evaluated prosthesis was minimal after the tests performed and with safety limits higher than those recommended by biomechanical engineering.

\section{Introdução}

O envelhecimento da população e a maior prevalência de pacientes com osteoartrose têm aumentado a frequência da indicação da artroplastia total do joelho (ATJ). ${ }^{1,2}$ A ATJ pode ser definida como um procedimento cirúrgico de alta complexidade para o tratamento da artrose capaz de demonstrar dados satisfatórios e duradouros na melhora da dor, da qualidade de vida, e da função do paciente, bem como na correção de deformidades e instabilidades de origens relacionadas a processos degenerativos que comprometem a articulação do joelho. ${ }^{3}$ Este procedimento apresenta excelentes resultados pós-operatórios em relação à sobrevida do implante, com índices de mais de $95 \%$ em pelo menos 10 anos de seguimento. ${ }^{4}$

O desgaste do polietileno pode produzir detritos que influenciam na soltura dos componentes protéticos. A ATJ com plataforma rotatória apresenta vantagens biomecânicas teóricas sobre um desenho de plataforma fixa. ${ }^{5}$ Estas vantagens incluem uma melhora da cinemática, aumentando a amplitude de movimento, facilitando a rotação axial, uma melhor distribuição das tensões entre os componentes femoral e tibial, e uma redução das forças de soltura na interface do implante com o osso. ${ }^{6-8}$ Muitas variáveis podem influenciar no comportamento de desgaste por atrito do polietileno, sendo elas o desenho da prótese, a matéria-prima utilizada, a técnica cirúrgica aplicada, e as morbidades do paciente, tais como o nível de atividade e a massa corpórea. 0 objetivo do presente estudo foi avaliar o desgaste do polietileno de uma prótese de joelho nacional ultracongruente de base rotatória (Rotaflex, Víncula, São Paulo, SP, Brasil).

\section{Materiais e Métodos}

Utilizou-se o método de ensaio com os parâmetros de carregamento e preparação citados na norma ISO 14243-1:2009Implants for Surgery - Wear of Total Knee Joint Prostheses - Part 1: Loading and Displacement Parameters for Wear Testing Machines with Load Control and Corresponding Environmental Conditions for Tests (Accredited) e os métodos de medição citados na norma ISO 14243-2:2009-Implants for Surgery Wear of Total Knee Joint Prostheses - Part 2: Methods of Measurement (Accredited). Para a avaliação do comportamento de desgaste, utilizou-se a prótese Rotaflex. ${ }^{9,10}$

Foram realizados 3 ensaios simultâneos no simulador de marcha da articulação de joelho ISO 14243-1 (EndoLab,
Riedering, Alemanha) em 5 sistemas, num total de 15 componentes (-Figuras $\mathbf{1}$ e $\mathbf{2}$ ).

Nas simulações, o implante foi fixado no dispositivo em extensão. Aplicou-se uma variação cíclica de flexo-extensão de $0^{\circ}$ a $58^{\circ}$ (-Tabela 1 ). Aplicou-se também uma força axial variando de $168 \mathrm{~N}$ a $2.600 \mathrm{~N}$, conforme o grau de flexão, simulando uma caminhada humana normal (- Tabela 1). A base tibial estava livre para se acomodar em relação ao componente femoral sob influência das forças de contato aplicadas, tendo este movimento todos os graus de liberdade, exceto o ângulo de flexo-extensão, que seguiu a variação cíclica especificada. Com esta simulação, as ações de força de contato aplicadas foram: força axial, força anteroposterior (AP, e torque de rotação tibial. Os componentes metálicos femoral e tibial, assim como o polietileno, foram imersos em um meio fluido simulando o líquido sinovial humano no decorrer de todo o ensaio, que foi realizado em um ambiente controlado, simulando as condições fisiológicas.

A avaliação do desgaste seguiu as normas ISO 14243-2:2009 com 10 milhões de ciclos e medições realizadas a cada milhão de ciclo. Seguindo as normas mencionadas, o desgaste foi avaliado analisando a perda da massa.

\section{Resultados}

A análise qualitativa da superfície do polietileno, após 10 milhões de ciclos, mostrou uma aparência de desgaste regular, com áreas polidas e foscas ( - Figura 2). Este padrão regular de desgaste indica uma estabilidade intrínseca dos componentes protéticos (- Figura $\mathbf{3}$ ).

$O$ resultado quantitativo do desgaste de massa por cada milhão de ciclo encontra-se na - Tabela 1. A - Figura 3 expressa os resultados mencionados na - Tabela 2.

A taxa média de desgaste foi de $2,56 \mathrm{mg}$ por milhão de ciclos, e foi determinada após 10 milhões de ciclos ( - Figura 1 ).

\section{Discussão}

A ATJ visa promover o alívio da dor e a melhora da função de maneira duradoura. Porém, a cirurgia pode falhar por uma série de razões, como soltura dos componentes, infecção, instabilidade e dor persistente, por exemplo. ${ }^{11}$ Visando a diminuição do desgaste do polietileno e, consequentemente, a produção de detritos, criou-se um componente tibial com plataforma rotatória, no qual o polietileno pode se 


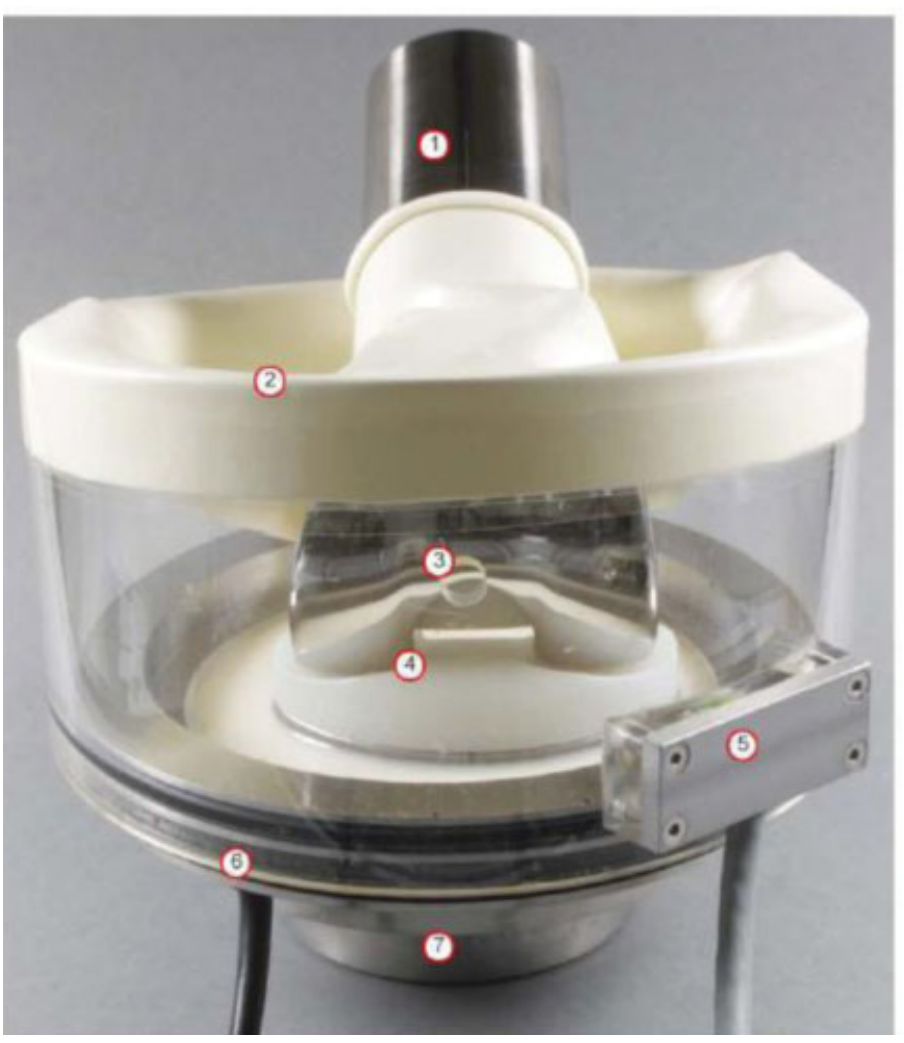

1- Dispositivo de fixação superior;
2- Dispositivo de isolamento do sistema para
exclusão de particulas externas da câmara;
3- Furo para preenchimento do fluido de teste
(após o preenchimento o furo é selado);
4- Sistema para ATJ;
5- Sensor de temperatura e nivel do fluido;
6- Aquecimento;
7- Dispositivo de fixação inferior.

Fig. 1 Representação da câmara de teste individual.

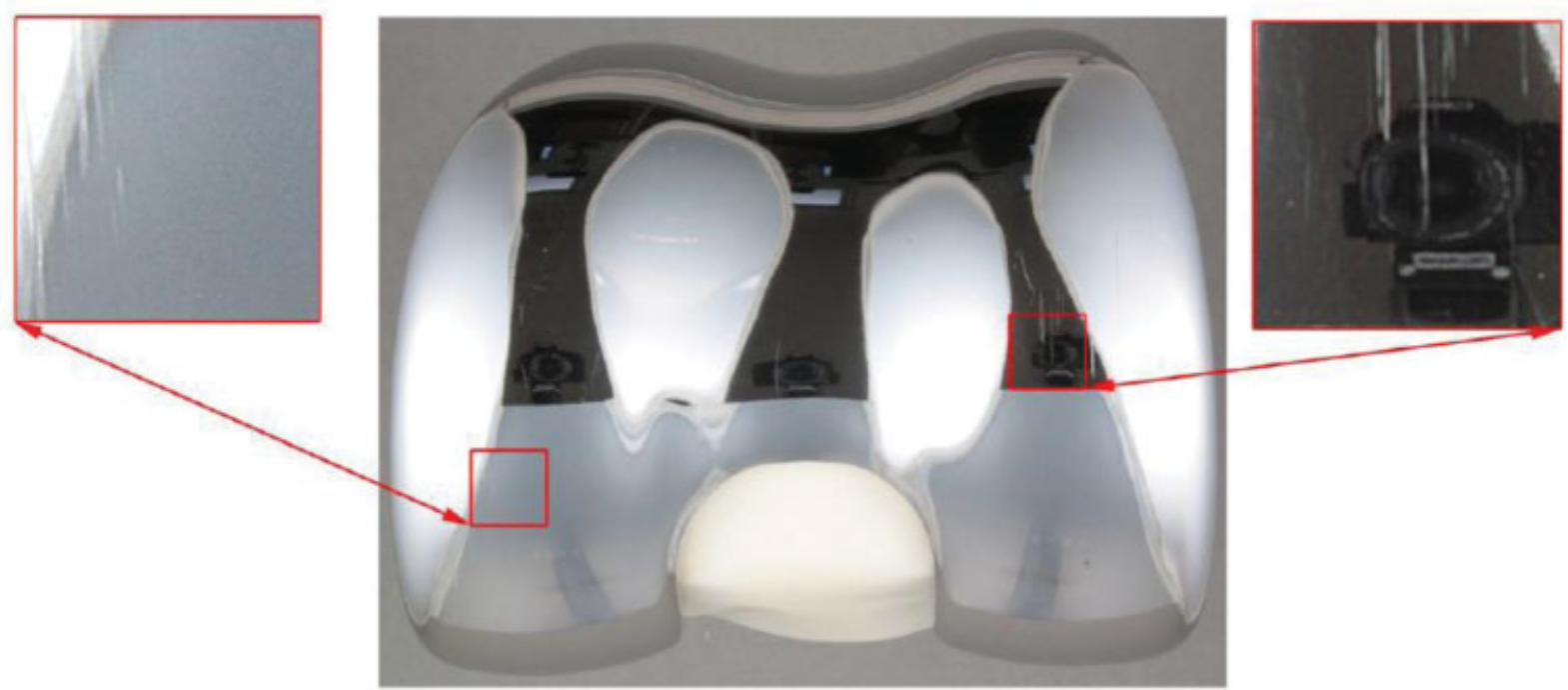

Fig. 2 Análise virtual qualitativa do desgaste.

movimentar rotacionalmente sobre o componente tibial, hipoteticamente diminuindo o atrito e o desgaste do componente. ${ }^{6,7,12}$

Em um estudo, ${ }^{11}$ os autores afirmam que as próteses com apoio rotatório ultracongruente apresentam a vantagem de uniformizar as pressões de contato entre os componentes, reduzindo assim a formação de partículas de polietileno e, consequentemente, a osteólise, além de adaptação melhor do mecanismo extensor às possíveis imperfeições no posicionamento rotacional do componente tibial. ${ }^{11} \mathrm{Um}$ estudo videofluoroscópico in vivo, seguido de reconstrução tridimensional das imagens obtidas, comparando-se próteses com base fixa e móveis, com a mesma origem e desenho, demonstrou que a superfície de contato femoro-tibial é duas vezes maior nas próteses com apoio rotatório quando comparadas às com apoio fixo. ${ }^{13}$ Nesse estudo, ${ }^{13}$ os autores notaram que os bons resultados foram semelhantes em ambos os modelos, mas, tanto objetivamente quanto subjetivamente, o apoio móvel foi julgado como sendo o mais aproximado do joelho normal. ${ }^{13}$ 


\section{Valores de desgaste $(\mathrm{mg} / \mathrm{mc})$}

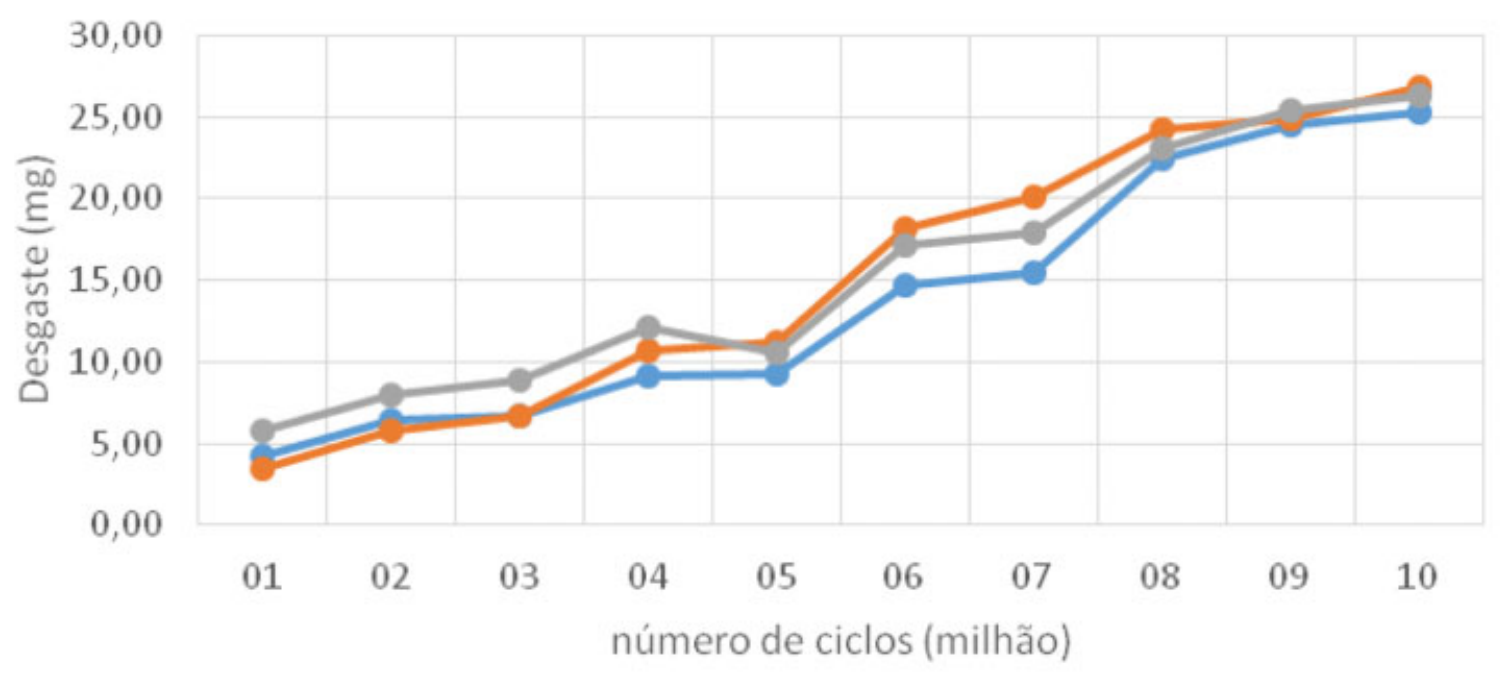

Acomplamento 1.1

Fig. 3 Desgaste do inserto X-UHMWPE versus número de ciclos.

Tabela 1 Parametros de carregamento

\begin{tabular}{|l|l|}
\hline Parâmetro & Valores conforme ISO 14243-1 \\
\hline Flexão/Extensão & $0^{\circ}$ a $58^{\circ}$ \\
\hline Força axial & $168 \mathrm{~N}$ a $2600 \mathrm{~N}$ \\
\hline Força anteroposterior & $-265 \mathrm{~N}$ a $110 \mathrm{~N}$ \\
\hline Torque & $-1 \mathrm{Nm}$ a $6 \mathrm{Nm}$ \\
\hline Frequencia & $1 \mathrm{~Hz}$ \\
\hline Fluido de teste & Soro de bezerro \\
\hline $\begin{array}{l}\text { Restrição de movimento - anteroposterior } \\
\text { (contrário ao movimento positivo anteroposterior) }\end{array}$ & $9,3 \mathrm{~N} / \mathrm{mm}$ \\
\hline $\begin{array}{l}\text { Restrição de movimento - anteroposterior } \\
\text { (contrário ao movimento negativo anteroposterior) }\end{array}$ & $44 \mathrm{~N} / \mathrm{mm}$ \\
\hline Restrição de rotação da tíbia** & $0,36 \mathrm{Nm} /{ }^{\circ}$ \\
\hline
\end{tabular}

Notas: *A restrição de movimento anteroposterior do sistema é 0 quando a articulação de joelho total é igual ou próximo a 2,5 mm em qualquer direção da posição de referência. ${ }^{* *} A$ restrição de rotação tibial do sistema é 0 quando a articulação de joelho total é igual ou próximo a $+/-6^{\circ}$ em qualquer sentido a partir do ponto de referência.

A alta durabilidade das próteses com componente de polietileno rotatório é bem elucidada na literatura, e as próteses podem atingir sobrevida superior a 20 anos em $97,7 \%$ dos casos. ${ }^{14}$

Schmidt et al ${ }^{15}$ estudaram a taxa de desgaste do polietileno em diferentes modelos de próteses já comercializados e consagrados no mercado, e encontraram valores de desgaste volumétrico que variaram de $1,9 \mathrm{mg} / \mathrm{mc}$ a $14,6 \mathrm{mg} / \mathrm{mc}$. No presente estudo, o resultado obtido foi de $2,67 \mathrm{mg} / \mathrm{mc}$ do desgaste volumétrico após 10 milhões de ciclos de ensaio, e, comparado aos valores de Schmidt et al, ${ }^{15}$ demonstrou-se que o sistema Rotaflex se aproxima à menor taxa encontrada $(1,9 \mathrm{mg} / \mathrm{mc})$. Além disso, o resultado mensurado do desgaste do componente de polietileno da prótese nacional avaliada, de $2,67 \mathrm{mg} / \mathrm{mc}$, obteve um desempenho de resistência ao desgaste 5,47 vezes superior aos valores máximos publicados.

\section{Conclusão}

O desgaste do polietileno da prótese avaliada foi mínimo após os ensaios realizados, e com os limites de segurança superiores aos preconizados pela engenharia biomecânica. 
Tabela 2 Dados de desgaste dos insertos X-UHMWPE (de polietileno) testados

\begin{tabular}{|c|c|c|c|c|c|c|}
\hline \multirow{3}{*}{$\begin{array}{l}\text { Acoplamento } \\
\text { Ciclos } \\
\text { (milhões) }\end{array}$} & \multicolumn{2}{|l|}{1.1} & \multicolumn{2}{|l|}{1.2} & \multicolumn{2}{|l|}{1.3} \\
\hline & \multicolumn{2}{|l|}{ Massa } & \multicolumn{2}{|l|}{ Massa } & \multicolumn{2}{|l|}{ Massa } \\
\hline & $\begin{array}{l}\text { Inserto } \\
\text { X-UHMWPE } \\
1.1 \text { (g) }\end{array}$ & $\begin{array}{l}\text { Inserto } \\
\text { X-UHMWPE } \\
1.1 \text { (mg) }\end{array}$ & $\begin{array}{l}\text { Inserto } \\
\text { X-UHMWPE } \\
1.2(\mathrm{~g})\end{array}$ & $\begin{array}{l}\text { Inserto } \\
\text { X-UHMWPE } \\
1.2 \text { (mg) }\end{array}$ & $\begin{array}{l}\text { Inserto } \\
\text { X-UHMWPE } \\
1.3 \text { (g) }\end{array}$ & $\begin{array}{l}\text { Inserto } \\
\text { X-UHMWPE } \\
1.3 \text { (mg) }\end{array}$ \\
\hline 0,0 & 44,71242 & 0,00 & 44,48231 & 0,00 & 44,66329 & 0,00 \\
\hline 0,5 & 44,70981 & 3,91 & 44,48121 & 2,41 & 44,65973 & 4,86 \\
\hline 1,0 & 44,71073 & 4,24 & 44,48138 & 3,48 & 44,66000 & 5,84 \\
\hline 2,0 & 44,71047 & 6,47 & 44,48102 & 5,81 & 44,65979 & 8,01 \\
\hline 3,0 & 44,71210 & 6,71 & 44,48202 & 6,69 & 44,66085 & 8,83 \\
\hline 4,0 & 44,71149 & 9,18 & 44,47986 & 10,70 & 44,65949 & 12,06 \\
\hline 5,0 & 44,71378 & 9,30 & 44,48183 & 11,14 & 44,66343 & 10,52 \\
\hline 6,0 & 44,71097 & 14,74 & 44,47747 & 18,13 & 44,65948 & 17,09 \\
\hline 7,0 & 44,71004 & 15,47 & 44,47531 & 20,08 & 44,65842 & 17,95 \\
\hline 7,5 & 44,71357 & 17,77 & 44,47810 & 23,13 & 44,66167 & 20,54 \\
\hline 8,0 & 44,70607 & 22,38 & 44,47417 & 24,17 & 44,65625 & 23,06 \\
\hline 9,0 & 44,70556 & 24,50 & 44,47504 & 24,90 & 44,65551 & 25,40 \\
\hline 10,0 & 44,70660 & 25,23 & 44,47497 & 26,75 & 44,65645 & 26,24 \\
\hline
\end{tabular}

Conflito de Interesses

Os autores declaram não haver conflito de interesses.

\section{Referências}

1 Jämsen E, Jäntti P, Puolakka T, Eskelinen A. Primary knee replacement for primary osteoarthritis in the aged: gender differences in epidemiology and preoperative clinical state. Aging Clin Exp Res 2012;24(06):691-698

2 Hernandez AJ, Camanho GL, Pécora JR. Artrodese do joelho: gênese e soluções. São Paulo: Atheneu; 2010

3 Ferreira MC, Oliveira JCP, Zidan FF, Franciozi CEDS, Luzo MVM, Abdalla RJ. Total knee and hip arthroplasty: the reality of assistance in Brazilian public health care. Rev Bras Ortop 2018;53(04):432-440

4 McLaughlin JR, Lee KR. Hybrid total knee arthroplasty: 10- to 16year follow-up. Orthopedics 2014;37(11):e975-e977

5 Tirico LEP, Pasqualin T, Pécora JO, Gobbi RG, Pécora JR, Demange MK. Estudo da estabilidade dos componentes na artroplastia total do joelho sem cimento. Acta Ortop Bras 2012;20(04):230-234

6 Kim YH, Park JW, Kim JS, Kulkarni SS, Kim YH. Long-term clinical outcomes and survivorship of press-fit condylar sigma fixedbearing and mobile-bearing total knee prostheses in the same patients. J Bone Joint Surg Am 2014;96(19):e168

7 Fransen BL, van Duijvenbode DC, Hoozemans MJM, Burger BJ. No differences between fixed- and mobile-bearing total knee arthroplasty. Knee Surg Sports Traumatol Arthrosc 2017;25(06):1757-1777

8 Cobra H, Palma IM. Polietileno tibial móvel na artroplastia total do joelho. Rev Bras Ortop 2009;44(06):475-478
9 ISO 14243-1:2009 Implants for surgery - Wear of total knee-joint prostheses-Part 1: Loading and displacement parameters for wear-testing machines with load control and corresponding environmental conditions for test. Disponível em: https://www. iso.org/standard/44262.html

10 ISO 14243-2:2009 Implants for surgery-Wear of total knee-joint prostheses-Part 2: Methods of measurement. Disponivel em: https://www.iso.org/standard/44263.html

11 Guglielmetti LG, Couto RC, Camargo OP, et al. Artroplastia total do joelho com o apoio tibial móvel. Avaliação dos resultados a médio prazo. Acta Ortop Bras 2010;18(06):310-314

12 Jorgensen NB, McAuliffe M, Orschulok T, Lorimer MF, de Steiger R. Major Aseptic Revision Following Total Knee Replacement: A Study of 478,081 Total Knee Replacements from the Australian Orthopaedic Association National Joint Replacement Registry. J Bone Joint Surg Am 2019;101(04):302-310

13 Ranawat CS, Flynn WF Jr, Saddler S, Hansraj KK, Maynard MJ. Long-term results of the total condylar knee arthroplasty. A 15year survivorship study. Clin Orthop Relat Res 1993;(286):94-102

14 McEwen HM, McNulty DE, Auger DD, et al. Wear analysis of mobile bearing knee. In: Hamelynck KJ, Stiehl JB, editors. LCS mobile bearing knee arthroplasty: a 25 years worldwide review. Heidelberg, Germany: Springer Verlag; 2002:67-73

15 Scmidt R, Jinnah R, Green J, Moseley J, Brownhill J. In vitro assessment of a cruciate retaining and cruciate sacrificing medially pivoting knee replacement. In: Annual Meeting of the Orthopaedic Research Society 2011, Long Beach, CA, 2011. (Poster $\mathrm{n}^{\circ}$ 1150. ORS 2011) 